\title{
Review of: "Hospitalizations and emergency department visits trends among elderly individuals in proximity to death: a retrospective population-based study"
}

Tatyana Mollayeva ${ }^{1}$

1 University of Toronto

Potential competing interests: The author(s) declared that no potential competing interests exist.

The authors have performed a secondary analysis of the electronic health records of individuals aged 65 and above, living in the Friuli-Venezia Giulia region of northeastern Italy, covering the period from 20002014, investigating hospitalizations and emergency department visits (EDV) two years preceding death in people $(n=142,834)$ who died between 2000 and 2014. Death was defined using death certificates. Residents who were "not continuously registered" in the region during the two years before dying ( $n=19,291$ individuals) were excluded.

Researchers estimated the percentage of one or more hospitalization or EDV two-year preceding death, stratified results by age group [65-74 years], [75-84 years], [85-94 years], and [>=95 years]), by sex, the leading cause of death (cancer, cardiovascular disease, respiratory disease). They also reported on other outcomes, including the duration of hospitalizations (please refer to the outcome section).

Authors reported that (1) percentage of individuals accessing [acute?] healthcare services increased exponentially in proximity to death (hospitalizations $=4.7$ months, EDVs $=3.9$ months before death); this was inversely related to age, with changes among the youngest and eldest decedents at 6.6 and 3.5 months for hospitalizations and at 4.6 and 3.3 months for EDVs, respectively; (2) healthcare use among individuals with cancer increased "earlier in life" (hospitalizations $=6.8, \mathrm{EDVs}=5.8$ months before death); (3) individuals with respiratory diseases were most likely to access hospital-based services during the last month of life; and (4) no sex-based differences were found. Researchers concluded that "greater use of acute healthcare services among younger individuals and cancer patients suggests that policies potentiating primary care support targeting these at-risk groups may reduce pressure on hospital-based services."

The strength of the data utilized in this study includes its population relevance and the ability to examine a range of hospitalizations and EDV, covering approximately $2 \%$ of the population of Italy (i.e., 1.2 mil people live in the Friuli-Venezia Giulia region). Likewise, there was a possibility to compare individuals across the age categories and biological sexes and the cause of death. The results are important for surveillance and public health. Below are some points that limit appreciation of the study results as they concern study methodology and reporting: 
1. Cohort selection "The main characteristics considered in our analyses among individuals included and excluded from the study were similarly distributed (data not shown)". The statement "data not shown" is unexpected in a study concerning epidemiological trends that aims to inform policy provision.

Reporting such information is required by published guidelines on observational studies (doi: https://doi.org/10.1136/bmj.39335.541782.AD).

2. Since the study outcomes concern repeated events (one or more visits) across ages, it is important to know how researchers ascertained that they did not double count events (https://doi.org/10.1093/ije/dyu222) of individuals who entered through ED and then were hospitalized after ED. Such information is not reported by researchers.

3. Some wording issues observed in the ICD-9-CM codes for main causes of death (cardiovascular disease, cancer). The codes reported as "cardiovascular disease (390-459)" are codes of diseases of the circulatory system; "cancer" are neoplasm codes, which also include benign neoplasms (210-229)http://www.icd9data.com/2012/Volume1/140-239/default.htm). Additional concern refers to selection of the three chapters out of 17 available within ICD-9-CM, rationale for which expects to be provided in the manuscript.

4. The rationale for using indicators of "triage color tag" in the sensitivity analyses is justified. However, results are not presented or discussed in the manuscript and reported in Supplementary figure 3, by sex and age, but not the main cause of death.

5. Introduction section expects to clearly state hypotheses regarding hospitalizations and EDV by sex, age, and cause of death. If omitted, this research should be designated as exploratory (http://dx.doi.org/10.4135/9781412953948.n219), and the discussion section then cannot discuss relevance to policy.

6. The abstract and introduction section starts by referring to COVID-19; this felt somewhat misleading to the reader, as the study period covered a period of 2010-2014. A statement like follows "No relevant changes occurred in the access to hospice, palliative care, long-term care or primary care services between 2015 and the outbreak of COVID-19" expects to be supported with evidence. Similar concerns apply to "Most studies on healthcare utilization at the end of life have focused on shorter periods ( 1 to 6 months) or longer periods considered in their entirety ( 1 to 2 years), thereby mitigating the strong timedependent differences within this timeframe." In the absence of citations provided to support the statements, the reader may question their validity.

7. The outcome list is extensive and includes (1) duration of hospitalization episodes; (2) the proportion of hospitalized subjects was then calculated for each month; (3) the number of hospitalizations calculated for each month; (4) the proportion of individuals with $\geq 1$ EDV; and (5) overall proportion of hospitalized subjects and those who accessed the emergency department during the last 24 months, 12 months, 6 months, and 1 month of life. With such an extensive list, the reader expected to see an extensive Statistical analyses section, which was limited to stating that "Nonlinear regression models were fitted to the monthly proportion of hospitalized patients and EDVs, to estimate change points" and a little 
explanation on the conditions imposed on the model concerning the change point analysis. No results have been provided to show whether these assumptions were satisfied in the current study. This is an important omission, as the methodology of the analysis is complex (https://variation.com/wpcontent/uploads/change-point-analyzer/change-point-analysis-a-powerful-new-tool-for-detectingchanges.pdf)

8. The list of exposures, predictors, potential confounders and effect modifiers of hospitalizations and EDV is not provided. Likewise, the list of variables with missing data, the percent of missing data, and how the missing data was handled is not reported (https://doi.org/10.2147/CLEP.S266428). If there were none, this expected to be clearly stated.

9. Overall, the statistical analysis section of the manuscript is very limited in providing important details. In particular, the reader would benefit from learning how models' selection were determined, which variables were included in the models, the models' fit, how change points were selected, and other specifics of the time point analysis.

In summary, while the research might be novel because it applied a change-point analysis to populationbased data, its written structure is far from optimal. The limited transparency in reporting steps in the statistical analysis, inability to replicate study results because the data cannot be shared publicly limits the appreciation and utility of the results. Finally, while "No informed consent or ethics committee approval was required because record linkage in this study was based on computerized databases of medical records with all the data being anonymized before analyses" might be an acceptable practice in research using administrative healthcare data (doi: 10.1016/j.ahj.2009.03.023), ethics committees also serve to help researchers to ensure that their research questions, hypotheses, research methodology, ability to answer research question with the proposed design, data linkage and storage, and sharing agreements all corresponds to best international practices in research design and reporting using human data. 